\title{
Monoclonal Antibody Targeting Neutralizing Epitope on H5N1 Influenza Virus of Clade 1 and 0 for Specific H5 Quantification
}

\author{
Fang $\mathrm{He}^{1}$ and Jimmy Kwang ${ }^{1,2}$ \\ ${ }^{1}$ Animal Health Biotechnology, Temasek Life Sciences Laboratory, National University of Singapore, Singapore 117604 \\ ${ }^{2}$ Department of Microbiology, Faculty of Medicine, National University of Singapore, Singapore 117597 \\ Correspondence should be addressed to Jimmy Kwang; kwang@tll.org.sg
}

Received 8 January 2013; Accepted 4 February 2013

Academic Editor: Prasert Auewarakul

Copyright (c) 2013 F. He and J. Kwang. This is an open access article distributed under the Creative Commons Attribution License, which permits unrestricted use, distribution, and reproduction in any medium, provided the original work is properly cited.

\begin{abstract}
H5N1 influenza viruses cause high mortality in avian and mammalian species, including humans. Antigenic drift in $\mathrm{H} 5$ sequence poses challenges in the development of vaccine and therapeutic antibody. In this study, a monoclonal antibody $11 \mathrm{G} 12$ was produced from inactivated H5N1 immunized mice. Results from IFA, ELISA, HI, and virus neutralization indicated that Mab 11 G12 can specifically recognize and neutralize $\mathrm{H} 5$ type hemagglutinin from clade 1 and 0 without any cross-reaction to any other clades of H5N1 viruses. Mab 11 G12 was used to differentiate and quantify the expression of H5N1 strain A/VietNam/1203/04 from a trivalent vaccine mix in ELISA. Sequencing of escape mutants identified that Mab 11G12 targets a major neutralizing epitope of influenza $\mathrm{H} 5$ hemagglutinin. The study indicated that some major neutralizing epitopes in H5s of early strains were mutated due to antigenic drift.
\end{abstract}

\section{Background}

Highly pathogenic avian influenza H5N1 virus has caused high mortality in birds and humans, raising concerns for the possibility of a future influenza pandemic [1]. In 1997, in Hong Kong, 18 humans were infected and 6 died in the first known case of $\mathrm{H} 5 \mathrm{~N} 1$ infecting humans $[2,3]$. Since the 2004 outbreaks of H5N1 influenza viruses from birds to human in Vietnam and Thailand, newly emerging avian influenza A viruses pose a continued lethal threat, not only to avian species but also to humans $[4,5]$. The H5N1 influenza viruses are currently divisible into 10 clades (0 to 9 ) on the basis of phylogenetic analysis of their hemagglutinin (HA) genes that have evolved in the A/Goose/Guangdong/96-like H5N1 lineage (Clade 0) [6]. Clade 0 includes all the early progenitors which are predominately strains in 1996-2002 from Hong Kong (HK) and China, while clade 1 includes human and bird isolates from Vietnam, Thailand, and Cambodia and bird isolates from Laos and Malaysia [7]. The human isolate A/VietNam/1203/04 (H5N1) from the 2004 outbreak was identified as the most pathogenic isolate in Clade $1.0[8,9]$. It was widely selected as the vaccine strain for H5N1. The stockpiling of a panel of vaccines with hemagglutinin (HA) antigenic variations, including A/VietNam/ 1203/2004, A/VietNam/1194/2004, A/Indonesia/05/2005, and $\mathrm{A} /$ Anhui/1/2005 vaccine viruses, was recommended by the WHO for vaccine development [7].

However, present vaccine strategies have been hindered by antigenic variation of the influenza strains. Immunity elicited with a single strain from a previous outbreak may not be able to provide sufficient protection against currently circulating H5N1 viruses [10]. Preventive measures against circulating $\mathrm{H} 5 \mathrm{~N} 1$ strains have received a lot of interest and effort globally to prevent another pandemic outbreak [11]. To overcome such limitations without any reduction in the vaccine efficacy and to completely realize the potential of vaccines worldwide, the concept of universal vaccines based on representative hemagglutinin mix has recently been proposed [12]. With an understanding in the distribution pattern of HA neutralizing epitopes, a trivalent $\mathrm{H} 5$ vaccine has been developed and proved to provide universal protection against multiple clades of $\mathrm{H} 5$ influenza viruses. The trivalent formulation includes H5N1 strains A/Indonesia/CDC669/2006 (clade 2.1), A/VietNam/1203/2004 (clade 1), and A/Anhui/1/05 
(clade 2.3) [13]. In order to specifically and individually identify and quantify HA expression of each strain, several strain-specific $\mathrm{H} 5$ monoclonal antibodies were selected and characterized (data not shown). In the present study, a monoclonal antibody $11 \mathrm{G} 12$ can specifically interact in IFA with H5 of the VN1203 strain without any cross-activity to either the Indonesia or Anhui strains. The reactivity was further evaluated in ELISA, HI, and virus neutralization against different clades of $\mathrm{H} 5 \mathrm{~N} 1$ viruses. The antigenic epitope for $11 \mathrm{G} 12$ was identified on H5 hemagglutinin. Results indicate that $11 \mathrm{G} 12$ is specific to $\mathrm{H} 5 \mathrm{~N} 1$ of clade 1.0 and 0.

\section{Materials and Methods}

2.1. Viruses and Cells. The $\mathrm{H} 5 \mathrm{~N} 1$ viruses used in these studies are from different clades (clade 0-A/HongKong/156/ 97, clade 1.0-A/HongKong/213/04, clade 1.0-A/VietNam/ 1203/04, clade 2.1.3-A/Indonesia/CDC669/06, clade 2.1.2A/Indonesia/CDC594/06, clade 2.2-A/Nigeria/6e/07, clade 2.2-A/chicken/Guangdong/178/04, clade 2.3-A/Anhui/1/ 05 , clade $2.3-\mathrm{A} / \mathrm{Jiangsu} / 2 / 07$, clade $4-\mathrm{A} /$ goose/Guiyang/ 337/06, clade 7-A/chicken/Shanxi/2/06 and clade 8-A/ chicken/Henan/12/04). Except for the two Indonesian strains, the remaining $\mathrm{H} 5$ influenza viruses were generated with reverse genetics in our lab as described previously [14]. Viruses were inoculated into the allantoic cavities of 11-dayold embryonated chicken eggs and harvested following $48 \mathrm{~h}$ of incubation at $37^{\circ} \mathrm{C}$. Virus titers were determined using hemagglutination assays according to standard methods [15]. $\mathrm{H} 5 \mathrm{~N} 1$ subtype viruses were inactivated with formaldehyde as described previously [16]. All experiments with live H5N1 subtype viruses were performed in a biosafety level 3 containment laboratory in compliance with $\mathrm{CDC} / \mathrm{NIH}$ and $\mathrm{WHO}$ recommendations and also were approved by the Agri-Food and Veterinary Authority and the Ministry of Health of Singapore.

MDCK cells were obtained from the American Type Culture Collection (ATCC). Cells were propagated in Dulbecco's minimal essential medium (DMEM) supplemented with 10\% fetal bovine serum. Virus stocks were grown in MDCK cells in DMEM supplemented with $0.5 \%$ bovine serum albumin (BSA) and $200 \mathrm{ng} / \mathrm{mL}$ of trypsin.

2.2. MAb Production. BALB/c mice were immunized twice subcutaneously at regular intervals of 2 weeks with inactivated whole virus from A/VietNam/1203/2004 at HA titer of $2^{8}$ in $0.1 \mathrm{~mL}$ of Phosphate Buffered Saline (PBS), which was emulsified with an equal volume of Montanide ISA 563 adjuvant (SEPPIC, France). Mice were boosted with the same viral antigen, 3 days before the fusion of splenocytes with SP2/0 cells. The fused cells were seeded in 96-well plates, and their supernatants were screened by immunofluorescence assays as described below. The hybridomas that produced the Mabs were cloned by limiting dilution at least three times. The positive Mabs were tested for their hemagglutination inhibition activity as described below. Immunoglobulins from selected positive Mabs were isotyped using a commercial isotyping kit (Amersham Bioscience, England) as described in the manufacturer's protocol, subcloned, and cultured. All animal experiments were carried out in accordance with the guides for Animal Experiments of the National Institute of Infectious Disease (NIID) and the "Animal Research: Reporting In Vivo Experiments" (ARRIVE) guidelines. Experiment protocols were reviewed and approved by Institutional Animal Care and Use Committee of the Temasek Life Sciences Laboratory (Project Approval nos. TLL-12-018 and TLL-12-015), National University of Singapore, Singapore.

2.3. Immunofluorescence Assay (IFA). MDCK cells cultured in 96-well plates were infected with AIV H5N1 strains. At 24$48 \mathrm{~h}$ postinfection, the cells were fixed with $4 \%$ paraformaldehyde for $30 \mathrm{~min}$ at room temperature and washed thrice with phosphate buffered saline (PBS), pH 7.4. Fixed cells were incubated with hybridoma culture supernatant at $37^{\circ} \mathrm{C}$ for $1 \mathrm{~h}$, rinsed with phosphate buffered saline (PBS), and then incubated with a 1:400 dilution of fluorescein isothiocyanate (FITC)-conjugated rabbit antimouse Immunoglobulin (Dako, Denmark). Cells were rinsed again in PBS, and antibody binding was evaluated by widefield epifluorescence microscopy (Olympus IX71).

2.4. Hemagglutination Inhibition Assay. Hemagglutination inhibition (HI) assays were performed as described previously [17]. Briefly, Mabs were serially diluted (2-fold) in V-bottom 96-well plates and mixed with $4 \mathrm{HA}$ units of $\mathrm{H} 5 \mathrm{~N} 1$ viruses. Plates were incubated for $30 \mathrm{~min}$ at room temperature, and $1 \%$ chicken RBCs were added to each well. The hemagglutination inhibition endpoint was the highest Mab dilution in which agglutination was not observed.

2.5. ELISA. 96-well, round-bottom microtiter plates (Nunc, Roskilde, Denmark) were coated with $1 \mathrm{ug} /$ well of capture MAb $11 \mathrm{G} 12$ in $100 \mathrm{uL}$ of carbonate buffer $(73 \mathrm{mM}$ sodium bicarbonate and $30 \mathrm{mM}$ sodium carbonate, $\mathrm{pH}$ 9.7) overnight at $4^{\circ} \mathrm{C}$ or $37^{\circ} \mathrm{C}$ for $2 \mathrm{~h}$. The plates were washed twice with PBST, followed by two washes with PBS after each incubation with antibody or antigen. The antibody-coated plates were blocked by incubation with $100 \mathrm{uL}$ of blocking buffer (PBS containing $5 \%$ milk) for $1 \mathrm{~h}$ at room temperature and then incubated at $37^{\circ} \mathrm{C}$ for $1 \mathrm{~h}$ with $100 \mathrm{uL}$ of virus-containing samples diluted in PBST. Virus binding was detected by incubation for $1 \mathrm{~h}$ at $37^{\circ} \mathrm{C}$ with $100 \mathrm{uL}$ of horseradish peroxidaseconjugated detection MAb $11 \mathrm{G} 12$ (800 ng) (in-house labeling; Roche). Chromogen development was mediated by the addition of $100 \mathrm{uL}$ of freshly prepared substrate solution (o-phenylenediamine-dihydrochloride; Sigma). The reaction was stopped by adding $0.1 \mathrm{~N}$ sulfuric acid, and the optical density at $490 \mathrm{~nm}$ was recorded. The detection limit was determined by the optical density value that gave a signal-tonoise ratio of 3 .

2.6. Microneutralization Assay. Neutralization activity of the monoclonal antibody against $\mathrm{H} 5 \mathrm{~N} 1$ strains was analyzed by microneutralization assay as previously described [18]. Briefly, ten times diluted Mab was further serially diluted (2fold) and incubated with $10050 \%$ tissue culture infectious 
doses (TCID50) of different clades of H5N1 strains for $1 \mathrm{~h}$ at room temperature and plated in duplicate onto MDCK cells grown in a 96-well plate. The TCID50 of each of the H5N1 strains in MDCK cell culture was determined by the Reed and Muench method. The neutralizing titer was assessed as the highest Mab dilution in which no cytopathic effect was observed by light microscopy.

2.7. Isolation and Analysis of Escape Mutants. The epitope recognized by Mab 11 G12 was mapped by characterization of escape mutants as described previously $[19,20]$. Briefly, $\mathrm{H} 5 \mathrm{~N} 1$ viruses were incubated with an excess of Mab for $1 \mathrm{~h}$ and then inoculated into 11-day-old embryonated chicken eggs. The eggs were incubated at $37^{\circ} \mathrm{C}$ for $24-48 \mathrm{~h}$. Virus was harvested and used for cloning in limiting dilution in embryonated chicken eggs, and the escape mutants were plaque purified. RNA was extracted from the allantoic fluid. The hemagglutinin gene was reverse transcriptase (RT)-PCR, amplified, and cloned into a TA-cloning vector (Promega), and several clones were sequenced. The sequences of individual clones were analyzed by comparison with the sequences of the parent virus.

\section{Results}

3.1. Characterization of Murine Monoclonal Antibody 11 G12. Mab $11 \mathrm{G} 12$ was produced from mice immunized with A/VietNam/1203/2004 H5N1 virus. 11G12 belongs to isotype IgM. $11 \mathrm{G} 12$ is able to detect H5 expression in MDCK cells infected with H5N1 strain A/VietNam/1203/2004 (VN1203) in IFA without any cross-reaction with uninfected MDCK cells. It presents neutralizing activity against VN1203 as indicated in HI and virus neutralization test. No activity was observed in Western blot with 11G12 against any H5 protein. All these findings suggest that Mab $11 G 12$ recognizes a neutralizing epitope in $\mathrm{H} 5$ of VN1203.

3.2. Specific Interaction with $\mathrm{H} 5$ Viruses in Clade 0 and 1 with 11G12. 11G12 was tested in IFA, HI, ELISA, and virus neutralization against various $\mathrm{H} 5$ strains besides VN1203. As shown in Figure 1, 11 G12 can only detect VN1203 and HK156 infection in IFA without any positive signals in MDCK cells infected with either CDC669 or Anhui strains. The results were confirmed in AC-ELISA (Figure 2). 11G12 can detect VN1203, HK156, and HK213 strains without any cross reaction to the rest of $\mathrm{H} 5$ strains tested. $\mathrm{In} \mathrm{HI}$ and $\mathrm{VN}$ test (Table 1), 11G12 succeeded in inhibiting VN1203, HK156, and HK213; all of which belong to either clade 0 or 1, while 11G12 failed to neutralize any virus strain from other clades tested.

3.3. Specific Quantify VN1203 Expression in Trivalent H5 Vaccines. A trivalent $\mathrm{H} 5$ vaccine was made from the mixture of H5 viruses including A/VietNam/1203/2004 (VN1203), A/Indonesia/CDC669/06, and A/Anhui/1/05. The three strains were individually tested in AC-ELISA to determine the binding curve with 11G12. AC-ELISA was further performed to identify and quantify VN1203 H5 expression in the trivalent mixture. As shown in Figure 3, 11 G12 showed
TABLE 1: HI and virus neutralization activity of Mab $11 G 12^{\mathrm{a}}$.

\begin{tabular}{lccc}
\hline Virus & Clade & HI titer & ${\text { VN } \text { titer }^{\mathrm{c}}}^{\mathrm{b}}$ \\
\hline A/HongKong/156/97 & 0 & 112 & 160 \\
A/VietNam/1203/04 & 1.0 & 256 & 320 \\
A/HongKong/213/2004 & 1.0 & 256 & 320 \\
A/Indonesia/CDC594/06 & 2.1 .2 & $<4$ & $<10$ \\
A/Indonesia/CDC669/06 & 2.1 .3 & $<4$ & $<10$ \\
A/Nigeria/6e/07 & 2.2 & $<4$ & $<10$ \\
A/chicken/Guangdong/178/04 & 2.2 & $<4$ & $<10$ \\
A/Anhui/1/05 & 2.3 & $<4$ & $<10$ \\
A/Jiangsu/2/07 & 2.3 & $<4$ & $<10$ \\
A/goose/Guiyang/337/06 & 4 & $<4$ & $<10$ \\
A/chicken/Shanxi/2/06 & 7 & $<4$ & $<10$ \\
A/chicken/Henan/12/04 & 8 & $<4$ & $<10$ \\
a Concentration of MAb at $0.5 \mathrm{mg} / \mathrm{mL}$. & & \\
b 4 HA unit of each virus strain used for HI. & \\
${ }^{c}$ One hundred TCID50 of each virus strain used for microneutralization \\
assays.
\end{tabular}

TABLE 2: Neutralizing epitopes of H5 HA using 11 G12 by escape mutations.

\begin{tabular}{lcccc}
\hline Parental virus & Nucleotide & $\begin{array}{c}\text { Nucleotide } \\
\text { change }\end{array}$ & $\begin{array}{c}\text { Amino } \\
\text { acid }\end{array}$ & $\begin{array}{c}\text { Amino acid } \\
\text { change }\end{array}$ \\
\hline A/VietNam/ & 454 & A to C & 152 & Lys to Gln \\
$1203 / 04$ & 464 & G to T & 155 & Ser to Ile \\
& 566 & A to T & 189 & Lys to Met \\
\hline
\end{tabular}

gradient reactivity against monovalent VN1203 at different HA titers, while negative signal was obtained against the rest two strains at all HA titers. The binding curve with trivalent H5 mix matched the one with mono-VN1203 at the same HA titers based on 11G12. At the meantime, the expression levels of Indonesia and Anhui $\mathrm{H} 5$ were quantified with the other two strain specific Mabs in the same method (data not shown).

3.4. Epitope Mapping for 11G12. Since Mab $11 \mathrm{G} 12$ is able to neutralize VN1203 H5N1 virus, the amino acids involved in forming the epitopes of Mab 11G12 were analyzed using selection of neutralization escape mutants. Point mutations in VN1203 H5 which abolish the neutralization by Mab $11 \mathrm{G} 12$ were identified. Sequencing of the complete HA genes isolated from 16 escape variants to $11 \mathrm{G} 12$ was performed. Six mutants were identified to carry single point mutations at amino acid positions 152 (Lys to Gln). Five strains mutate at 155 (Ser to Ile) and the other 5 strains at 189 (Lys to Arg) (excluding signal peptide) (Table 2).

\section{Discussion}

Generated from mice immunized with VN1203 H5N1 virus, $11 \mathrm{G} 12$ is able to recognize specifically $\mathrm{H} 5$ viruses from clade 0 and 1.0. The specific reactivity was observed in IFA, ELISA, and virus neutralization. 11G12 presented strong positive neutralizing reaction with all the tested viruses of clade 0 and 

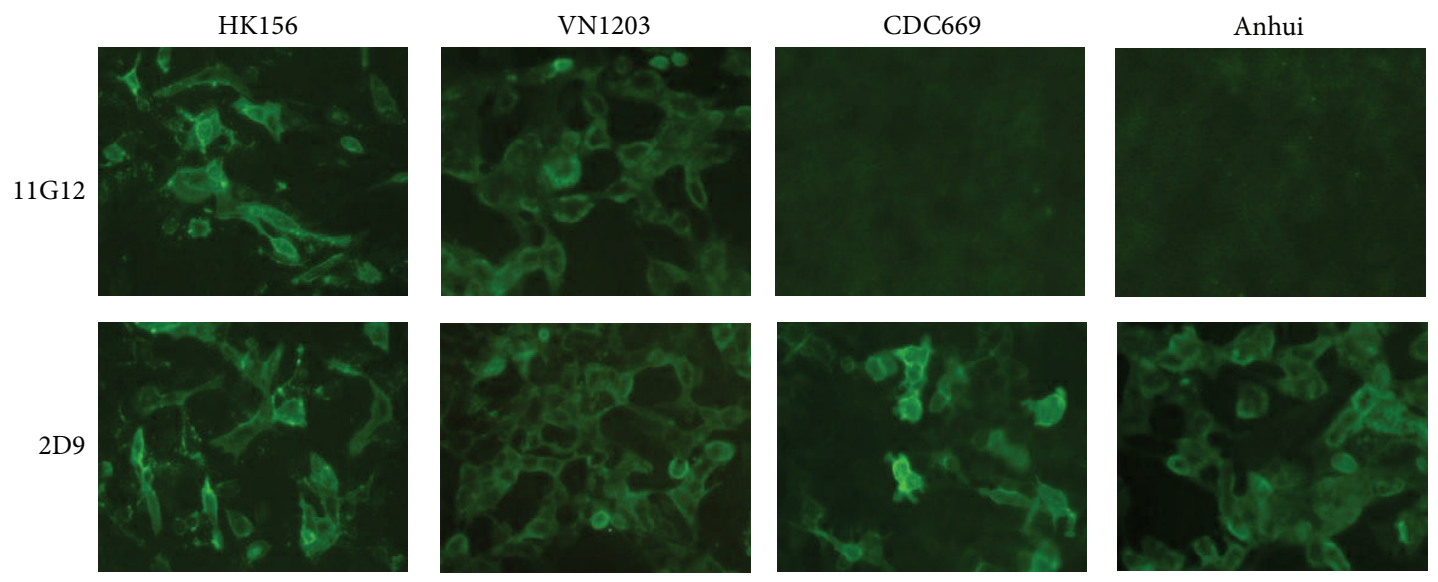

FIGURE 1: Reactivity against different H5 strains with $11 \mathrm{G} 12$ in H5 infected MDCK cells with IFA. MDCK cells were infected with H5 influenza virus as listed. Fixed cells were stained with either $11 \mathrm{G} 12$ or 2D9. 2D9 is a Mab with broad recognition to all these H5 strains used.

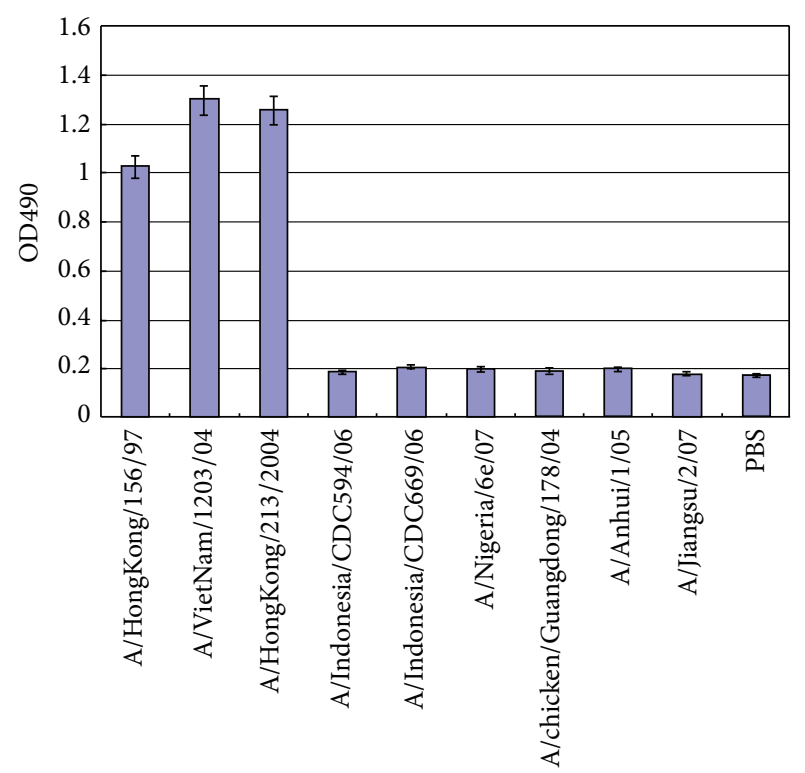

FIGURE 2: Reactivity against different $\mathrm{H} 5$ strains with $11 \mathrm{G} 12$ in ACELISA. $100 \mathrm{uL}$ of each virus at $2^{8} \mathrm{HA}$ titer was tested in the ELISA.

1.0, while $11 \mathrm{G} 12$ cannot react and neutralize any tested viruses from other clades. Mab $11 \mathrm{G} 12$ recognizes a neutralizing epitope containing at least 152Lys, 155Ser, and 189Lys on $\mathrm{H} 5$ hemagglutinin. The combination of three amino acids at these sites is mainly found in $\mathrm{H} 5$ influenza viruses from clade 0 and 1.0. These findings indicate that $11 \mathrm{G} 12$ recognizes a neutralizing epitope existing in early $\mathrm{H} 5$ strains, such as strains of clade 0 and 1.0. With mutations occurring in the antigenic structure during evolution, the affinity between 11 G12 and the epitope was abolished finally, indicating that antigenic drift does cause virus evasion from the immunity elicited by vaccination with early strains.

Mab 11 G12 presents the binding preference to the antigenic structures belonging to these $\mathrm{H} 5 \mathrm{~N} 1$ strains of clade

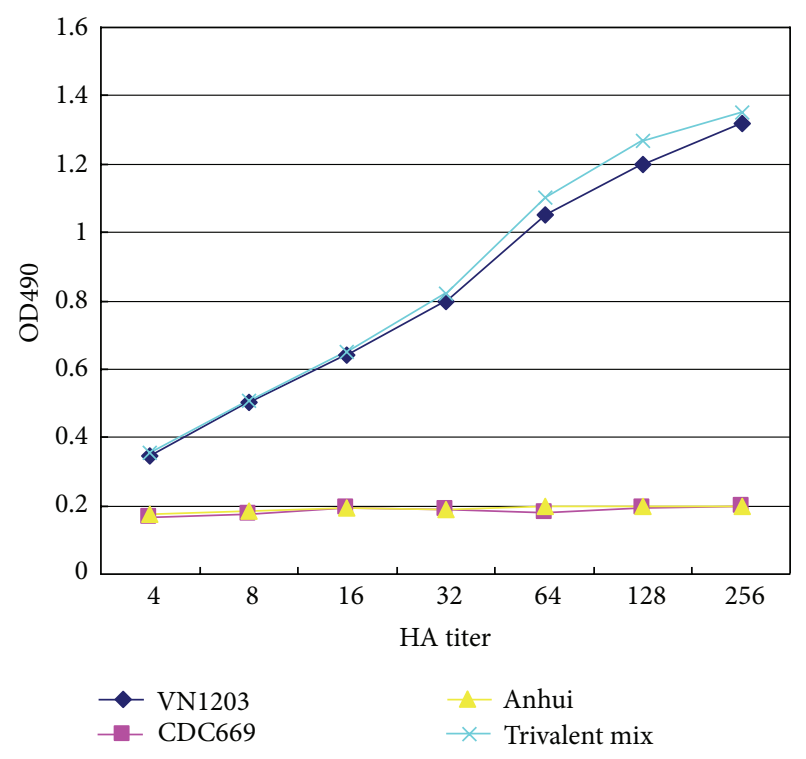

Figure 3: Binding curves with Mab 11 G12 and monovalent and trivalent $\mathrm{H} 5$ mix.

0 and 1.0. This finding not only provides a solid evidence for antigenic drift, but also emphasizes the current needs for a universal $\mathrm{H} 5$ vaccine, which evokes an application of $11 \mathrm{G} 12$ in vaccine production. The specificity of Mab $11 \mathrm{G} 12$ allows it to identify and quantify VN1203 HA expression specifically in the trivalent $\mathrm{H} 5$ vaccine. The trivalent $\mathrm{H} 5$ universal vaccine is based on three types of $\mathrm{H} 5 \mathrm{~s}$ from different clades, different years, and different countries. The universal vaccine formulation can be produced based on three $\mathrm{H} 5 \mathrm{~N} 1$ influenza viruses mixed. Besides, the $\mathrm{H} 5$ combination can be expressed in any other vaccine production platform, such as baculovirus [18] and Modified vaccinia Ankara. For the maximal efficacy of the trivalent vaccine, it is important to keep the three $\mathrm{H} 5 \mathrm{~s}$ at the same proportion in the vaccine 
formulation. Thus, Mab 11G12 serves as an ideal reagent to specifically determine VN1203 H5 expression. Together with other specific Mabs for the rest two H5s, Mab 11 G12 contributes to the quality control in vaccine production.

Besides, employment of $11 \mathrm{G} 12$ in $\mathrm{H} 5$ diagnosis provides ease in clade-specific detection. H5 positive samples identified in traditional $\mathrm{H} 5$ rapid tests, such as lateral flow, can be further tested with $11 \mathrm{G} 12$ whether they belong to clade 0 or 1.0. The tests could be finished within 30 minutes, which are more efficient and cost-effective than real time PCR. Since cross-clade protection may not be available from every $\mathrm{H} 5$ vaccine and treatment, it is important to identify the $\mathrm{H} 5$ clade infected and provide effective therapeutic Mabs accordingly, which can be humanized 11G12. Together with other cladespecific Mabs for the other H5s, Mab 11 G12 serves as a fine reagent for $\mathrm{H} 5$ clade-specific diagnosis.

\section{Conclusion}

Mab 11G12 can specifically recognize and neutralize H5 type hemagglutinin from clade 1 and 0 without any cross-reaction to any other clades of $\mathrm{H} 5 \mathrm{~N} 1$ viruses. The specificity of Mab 11 G12 not only allows it to identify and quantify VN1203 HA expression specifically in a universal trivalent $\mathrm{H} 5$ vaccine, but also gives a solid evidence for the phenomenon of antigenic drift.

\section{Conflict of Interests}

The authors declare that they have no conflict of interests.

\section{Authors' Contribution}

F. He and J. Kwang conceived and designed the experiments and analyzed the data. F. He performed the experiments and wrote the paper.

\section{Acknowledgment}

The authors are grateful for the financial support received from Temasek Life Science Laboratory, Singapore.

\section{References}

[1] S. Sambhara and G. A. Poland, "H5N1 avian influenza: preventive and therapeutic strategies against a pandemic," Annual Review of Medicine, vol. 61, pp. 187-198, 2010.

[2] A. S. Lipatov, Y. A. Smirnov, N. V. Kaverin, and R. G. Webster, "Evolution of avian influenza viruses H5N1 (1997-2004) in southern and south-eastern Asia," Voprosy Virusologii, vol. 50, no. 4, pp. 11-17, 2005.

[3] T. Yamada, A. Dautry, and M. Walport, "Ready for avian flu?" Nature, vol. 454, no. 7201, p. 162, 2008.

[4] F. He, Q. Du, Y. Ho, and J. Kwang, "Immunohistochemical detection of Influenza virus infection in formalin-fixed tissues with anti-H5 monoclonal antibody recognizing FFWTILKP," Journal of Virological Methods, vol. 155, no. 1, pp. 25-33, 2009.
[5] T. Horimoto, N. Fukuda, K. Iwatsuki-Horimoto et al., "Antigenic differences between H5N1 human influenza viruses isolated in 1997 and 2003," Journal of Veterinary Medical Science, vol. 66, no. 3, pp. 303-305, 2004.

[6] R. G. Webster and E. A. Govorkova, "H5N1 influenzacontinuing evolution and spread," New England Journal of Medicine, vol. 355, no. 21, pp. 2174-2177, 2006.

[7] P. Yang, Y. Duan, P. Zhang et al., "Multiple-clade H5N1 influenza split vaccine elicits broad cross protection against lethal influenza virus challenge in mice by intranasal vaccination," PLoS ONE, vol. 7, no. 1, Article ID e30252, 2012.

[8] H. L. Yen, J. R. Aldridge, A. C. M. Boon et al., "Changes in $\mathrm{H} 5 \mathrm{~N} 1$ influenza virus hemagglutinin receptor binding domain affect systemic spread," Proceedings of the National Academy of Sciences of the United States of America, vol. 106, no. 1, pp. 286291, 2009.

[9] H. L. Yen, A. S. Monto, R. G. Webster, and E. A. Govorkova, "Virulence may determine the necessary duration and dosage of oseltamivir treatment for highly pathogenic A/Vietnam/1203/04 influenza virus in mice," Journal of Infectious Diseases, vol. 192, no. 4, pp. 665-672, 2005.

[10] G. L. Chen and K. Subbarao, "Attacking the flu: neutralizing antibodies may lead to "universal" vaccine," Nature Medicine, vol. 15, no. 11, pp. 1251-1252, 2009.

[11] T. T. Y. Lam, C. C. Hon, O. G. Pybus et al., "Evolutionary and transmission dynamics of reassortant $\mathrm{H} 5 \mathrm{~N} 1$ influenza virus in Indonesia," PLoS Pathogens, vol. 4, no. 8, Article ID e1000130, 2008.

[12] C. A. Russell, T. C. Jones, I. G. Barr et al., "Influenza vaccine strain selection and recent studies on the global migration of seasonal influenza viruses," Vaccine, vol. 26, supplement 4, pp. D31-D34, 2008.

[13] M. Prabakaran, F. He, T. Meng et al., "Neutralizing epitopes of influenza virus hemagglutinin: target for the development of a universal vaccine against H5N1 lineages," Journal of Virology, vol. 84, no. 22, pp. 11822-11830, 2010.

[14] H. T. Ho, H. L. Qian, F. He et al., "Rapid detection of H5N1 subtype influenza viruses by antigen capture enzyme-linked immunosorbent assay using H5- And N1-specific monoclonal antibodies," Clinical and Vaccine Immunology, vol. 16, no. 5, pp. 726-732, 2009.

[15] A. N. Abdel-Ghafar, T. Chotpitayasunondh, Z. Gao et al., "Update on avian influenza A (H5N1) virus infection in humans," New England Journal of Medicine, vol. 358, no. 3, pp. 261-273, 2008.

[16] Q. He, S. Velumani, Q. Du et al., "Detection of H5 avian influenza viruses by antigen-capture enzyme-linked immunosorbent assay using H5-specific monoclonal antibody," Clinical and Vaccine Immunology, vol. 14, no. 5, pp. 617-623, 2007.

[17] R. G. Webster, Y. Kawaoka, J. Taylor, R. Weinberg, and E. Paoletti, "Efficacy of nucleoprotein and haemagglutinin antigens expressed in fowlpox virus as vaccine for influenza in chickens," Vaccine, vol. 9, no. 5, pp. 303-308, 1991.

[18] M. Prabakaran, S. Velumani, F. He et al., "Protective immunity against influenza $\mathrm{H} 5 \mathrm{~N} 1$ virus challenge in mice by intranasal co-administration of baculovirus surface-displayed HA and recombinant CTB as an adjuvant," Virology, vol. 380, no. 2, pp. 412-420, 2008.

[19] N. V. Kaverin, I. A. Rudneva, E. A. Govorkova et al., "Epitope mapping of the hemagglutinin molecule of a highly pathogenic H5N1 influenza virus by using monoclonal antibodies," Journal of Virology, vol. 81, no. 23, pp. 12911-12917, 2007. 
[20] N. V. Kaverin, I. A. Rudneva, N. A. Ilyushina et al., "Structure of antigenetic sites on the haeomagglutinin molecule of $\mathrm{H} 5$ avian influenza virus and phenotypic variation of escape mutants," Journal of General Virology, vol. 83, no. 10, pp. 2497-2505, 2002. 


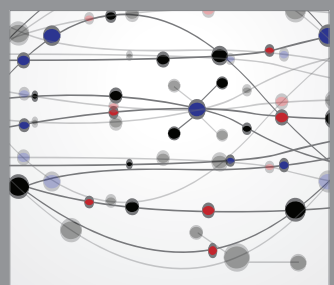

The Scientific World Journal
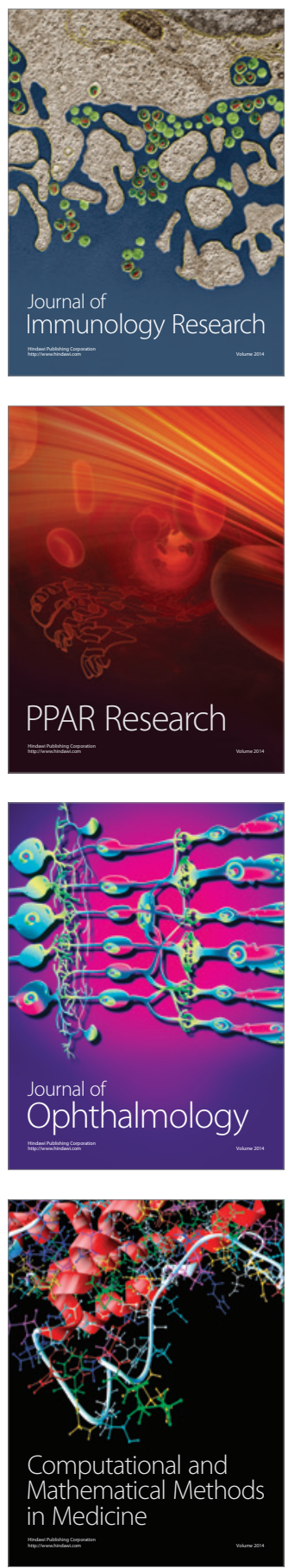

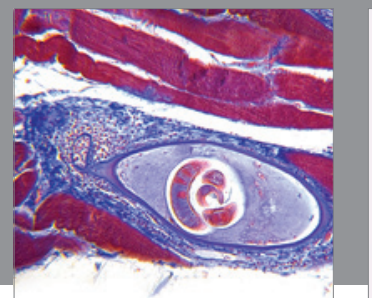

Gastroenterology

Research and Practice
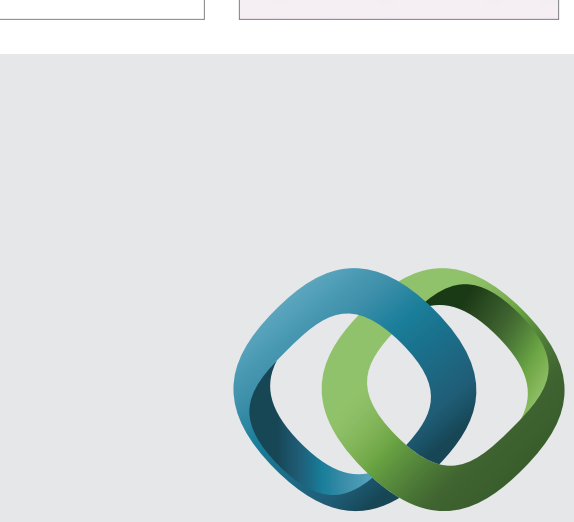

\section{Hindawi}

Submit your manuscripts at

http://www.hindawi.com
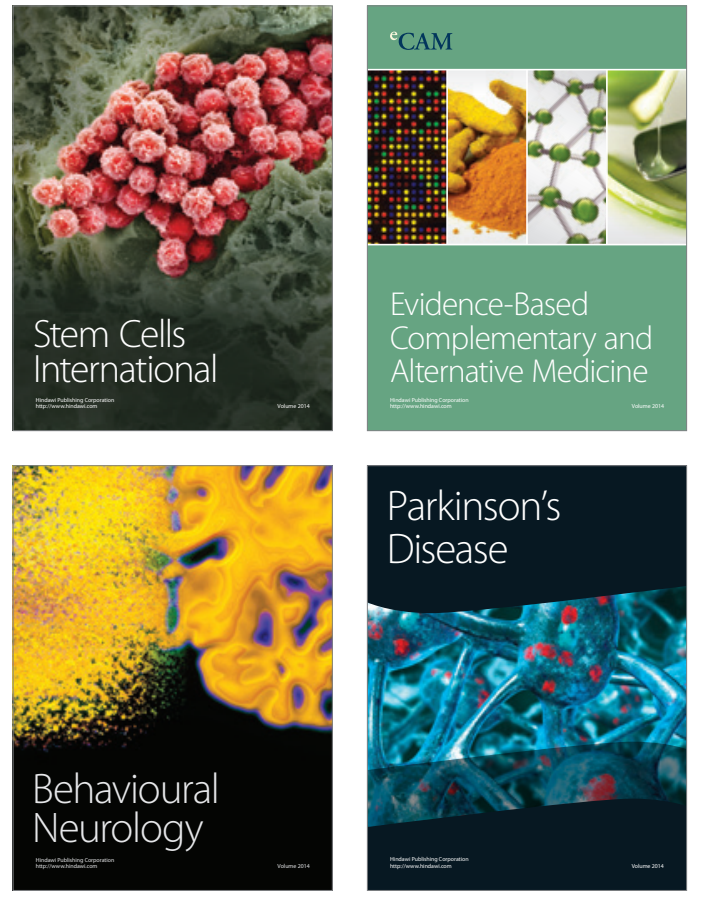
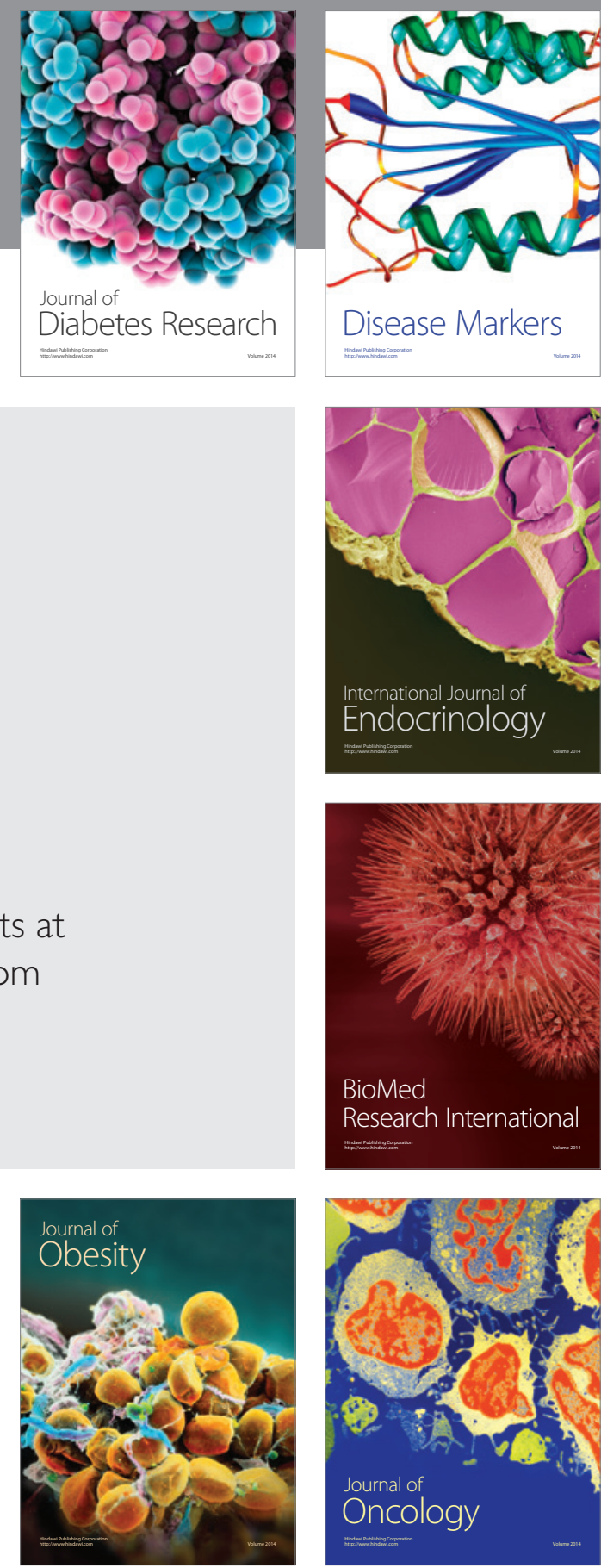

Disease Markers
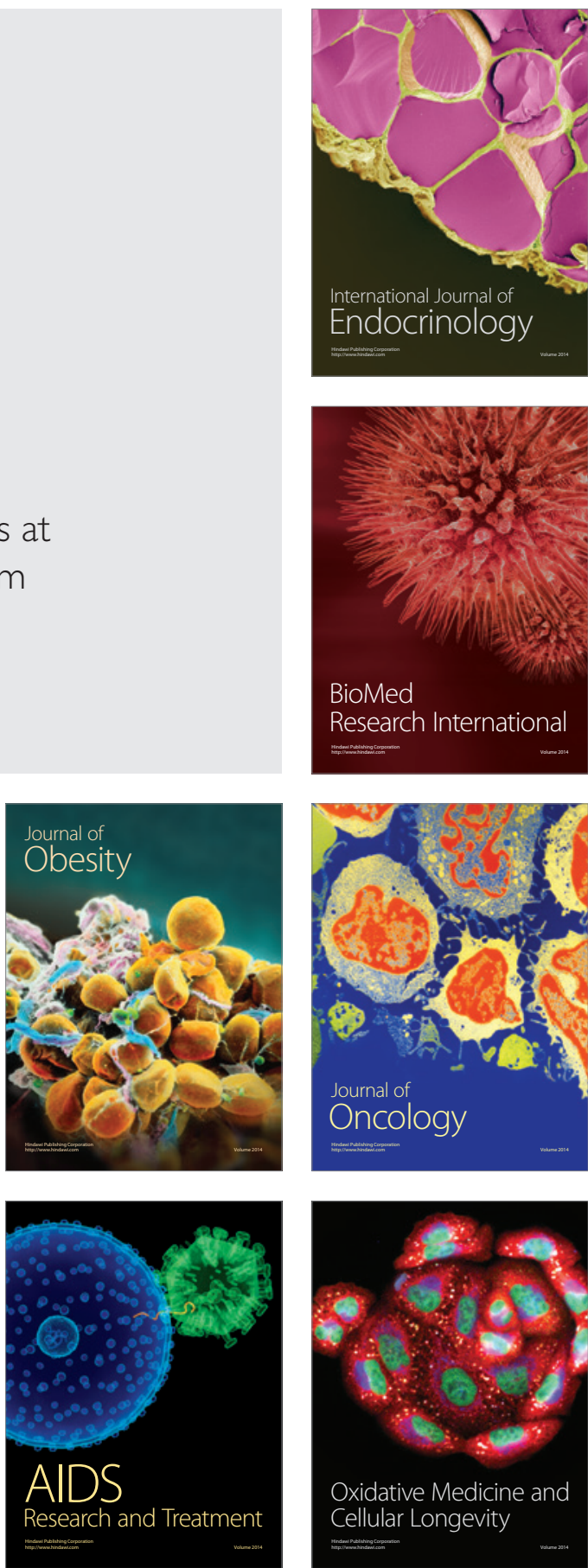